Musées, Patrimoine et Culture scientifiques et techniques

$165 \mid 2016$

mai-juin 2016

\title{
Le modèle du numérique, une voie nouvelle pour mettre en scène les savoirs?
}

\section{Ludovic Maggioni}

\section{OpenEdition \\ Journals}

Édition électronique

URL : http://journals.openedition.org/ocim/1654

DOI : $10.4000 /$ ocim. 1654

ISSN : 2108-646X

\section{Éditeur}

OCIM

\section{Édition imprimée}

Date de publication : 1 mai 2016

Pagination : 12-18

ISSN : 0994-1908

Référence électronique

Ludovic Maggioni, «Le modèle du numérique, une voie nouvelle pour mettre en scène les savoirs ? », La Lettre de I'OCIM [En ligne], 165 | 2016, mis en ligne le 01 mai 2017, consulté le 01 mai 2019. URL http://journals.openedition.org/ocim/1654; DOI : 10.4000/ocim.1654

Ce document a été généré automatiquement le 1 mai 2019.

Tous droits réservés 


\title{
Le modèle du numérique, une voie nouvelle pour mettre en scène les savoirs?
}

\author{
Ludovic Maggioni
}

L'exposition Confidences d'outre-tombe, squelettes en question a été présentée au musée Dauphinois de Grenoble jusqu'en janvier dernier.

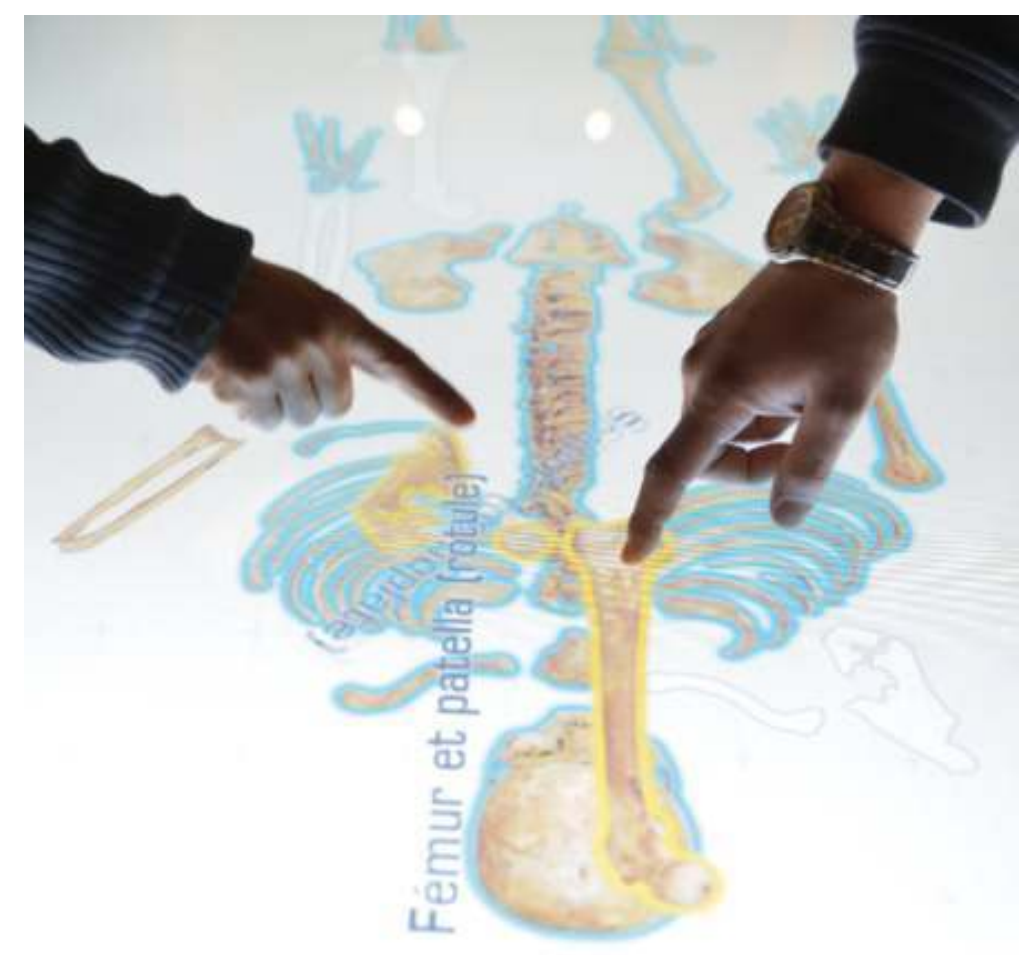

(c) Musée Dauphinois 
1 Les technologies du numérique en plein essor ces dernières années bouleversent les organisations sociales et individuelles. Les musées, centres de science et autres lieux culturels ne sont pas en reste et se posent également de nombreuses questions quant à ces changements. "Le numérique impose à la médiation d'évoluer, voire de changer ces modes. Selon une enquête du CSA réalisée pour l'Académie des sciences en septembre 2014 la science bénéficie d'une image positive chez les 15-25 ans, elle éveille en eux un "intérêt poli", surtout chez les filles. Les innovations technologiques et les objets numériques suscitent en revanche un vrai engouement"1.

2 Les manières de transmettre les savoirs évoluent : Wikipédia est devenu le 5e site le plus consulté tous sites confondus en septembre $2014^{2}$. Internet devient pour les jeunes la première source d'information et d'accès à la connaissance (Donnat, 2009).

3 Le monde de la culture et plus particulièrement les acteurs de la culture scientifique s'interrogent et expérimentent de nouvelles manières de dialoguer avec leurs publics, pour ce faire des expériences sont mises en place dans de nombreux lieux culturels comme dans l'exposition Confidences d'outre-tombe, squelettes en question présentée au musée Dauphinois à Grenoble du 20 décembre 2014 au 4 janvier 2016. Une exposition coproduite par le musée Dauphinois, le Centre de Culture Scientifique Technique et Industrielle de Grenoble - La Casemate, le musée archéologique Grenoble Saint-Laurent, l'INRAP et les Pompes funèbres de l'Isère.

Dans le cas de Confidences d'outre-tombe, squelettes en question l'intégration de dispositifs numériques dans l'exposition a été pensée selon deux grands axes : comment co-construire une exposition avec des visiteurs ? Comment le numérique peut-il révéler le patrimoine?

\section{Une exposition "co-construite" avec des visiteurs}

Le vent de la participation souffle sur le XXIe siècle, à la manière des médias numériques, certains acteurs de la culture souhaitent dialoguer d'une manière différente avec leurs audiences en écoutant et en leur donnant la parole. La prise de parole dans les musées n'a en soi rien de nouveau, de nombreux exemples, et en particulier au musée Dauphinois à Grenoble, pourraient être cités ${ }^{3}$. Ce qui change aujourd'hui, à l'ère du numérique, ce sont les modes de participation. Le modèle des Wiki et autres réseaux sociaux comme Facebook et Twitter permettent de renouveler les formes de dialogue avec les institutions. Ces nouvelles formes de participation peuvent être inspirantes pour la production de dispositifs numériques dans une exposition de science, tel a été l'enjeu du développement de l'exposition Confidences d'outre-tombe, squelettes en question. Pourrions-nous tous contribuer à la réalisation d'une exposition ? Faut-il être expert ? Les propos qui suivent permettent d'effectuer un retour sur une expérience où l'usager est placé au cœur de la production, un concept développé dans les Living Labs ${ }^{4}$ qui mettent en place des méthodologies croisant des approches d'idéations, de productions et de tests.

\section{Première étape : une séance de créativité}

6 Dans la veine de Museomix ${ }^{5}$, un évènement qui rassemble depuis cinq ans sur deux jours des passionnés de musées et de technologies pour inventer des dispositifs inédits de 
partage des savoirs, La Casemate avec ses partenaires a mis en place une expérience originale au cœur de son Living Lab : donner une parole scénographique à des visiteurs ${ }^{6}$.

\section{Retour sur une expérience originale}

7 Tout a commencé les 3 et 4 avril 2013. Vingt-deux personnes ayant répondu à un appel à participation lancé sur les réseaux de La Casemate et de ses partenaires se sont présentées à Ideas Lab sur le site du CEA pour imaginer la future exposition. Pendant 2 jours, ces personnes en formation, ces étudiants, ces passionnés de techniques, ces archéologues ont développé quatre scénarii originaux au sein de quatre équipes aux noms très évocateurs d'une ambiance improbable : "Os'mose", "Squelette Academy", "Squelette ville", "Squelettes antiques enquêtes en kit". Ils se sont impliqués très fortement dans cette expérience comme en témoigne Jérémie : "C'était sympa de travailler avec des scientifiques [ndlr : tous ne l'étaient pourtant pas]. J'ai apprécié d'échanger avec des personnes qui avaient d'autres idées. J'étais dans un groupe très productif et certaines de mes idées ont été reprises notamment le passage aléatoire d'un faux fantôme. Bref j'ai aimé, c'est à refaire !". Selon Samir : "On a travaillé sur deux idées : un guide virtuel en forme de squelette ou un vrai guide qui utiliserait les nouvelles technologies dans les pièces. Certaines idées étaient incroyables"7.

De ces deux journées construites avec un programme alternant présentation technologique avec la découverte en présence de start-up du showroom technologique du CEA, la visite de lieux culturels comme le musée archéologique de Grenoble, la rencontre avec un artiste réalisant du light painting et l'alternance de séances de créativité et de débriefing sont issues beaucoup d'idées, de projections en vrac : des brouillons, des schémas, des dessins plus ou moins aboutis permettant d'appréhender les représentations des futurs usagers de l'exposition. L'objectif de ces temps est de stimuler l'imaginaire des participants qui sont issus d'horizons différents, et après des séances de divergences d'association d'idées, de les faire converger sur leurs idées, ces idées qu'ils rêvent de voir se développer un jour dans une exposition.

Les participants prennent très au sérieux ce travail et sont très surpris lors des restitutions finales des propositions qui peuvent être faites à des professionnels. Au-delà du travail d'idéation qu'ils produisent pour l'exposition, ils découvrent également le travail en équipe et expérimentent des méthodes de créativité tout en rencontrant des professionnels. Ils se retrouvent au cœur des processus d'innovations ouvertes. Pour eux, ce type d'expérience pourra ensuite être valorisé sur les réseaux, dans leur CV par exemple, ils vivent un véritable moment professionnel ${ }^{8}$. 
Le dispositif "Strat'os" proposait une fouille numérique permettant au public de découvrir des objets virtuels en mettant en scène la gestuelle d'une fouille archéologique réelle.

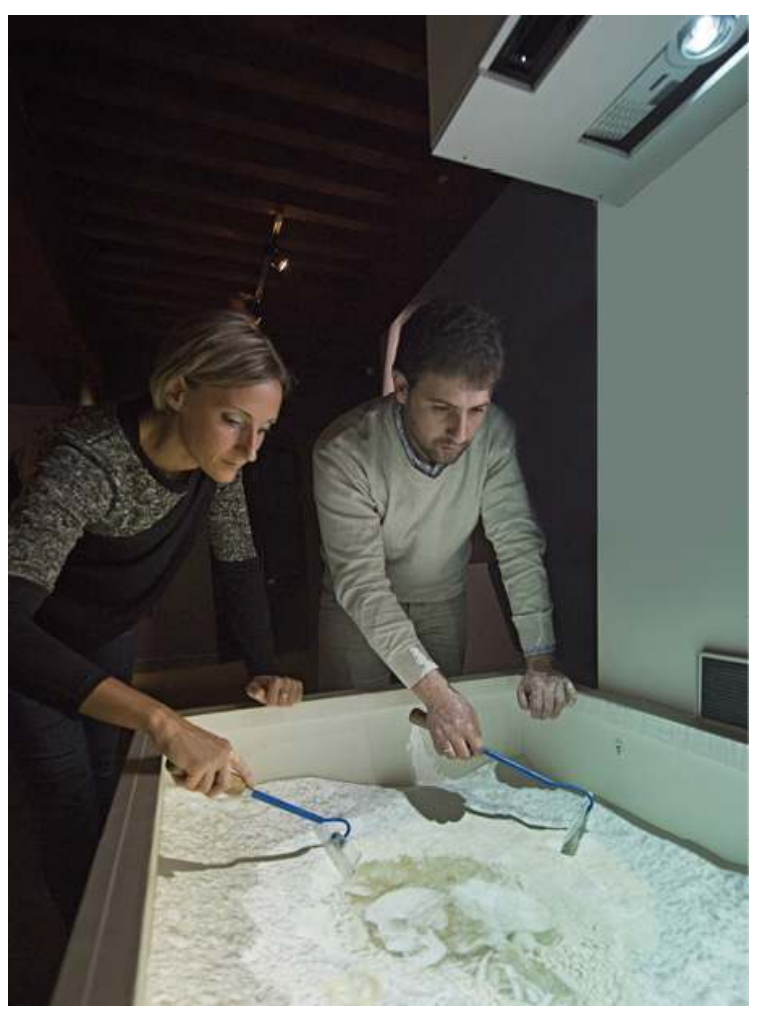

(c) Musée Dauphinois

\section{Deuxième étape : appropriation et intégration des idées dans le scénario d'exposition}

Les séances de créativité sont des moments de foisonnement, de nombreux concepts sont développés par les participants sans réelle limite, si ce n'est les présentations faites par les porteurs de projet. Le temps de l'appropriation et d'une éventuelle intégration arrive alors pour l'équipe de production. Il s'agit de mettre en place une grille d'analyse des projets proposés pour savoir s'ils sont pertinents pour l'exposition. Différents critères sont mis en place pour mener ce travail : quelles valeurs pour mettre en scène la compréhension des savoirs? Quels types d'interactivités sont proposés par les concepts? Quels dialogues s'instaurent avec le patrimoine ? Quels sont les possibilités de réalisation technologiques, est-ce réaliste techniquement, financièrement? Quelles idées générales sont présentes dans les projets proposés, sont-elles redondantes?

11 Dans cette phase chaque idée est prise au sérieux et étudiée selon ces critères. La redondance a été un élément important en se posant la question à chaque fois : est-ce une banalité qui n'a pas d'intérêt ou est-ce une attente des participants ? Cinq types de dispositifs ont ainsi été retenus. Ils ont été mis à l'épreuve du scénario de l'exposition et ont vu leur contenu s'enrichir. 


\section{Réalisation de cinq dispositifs numériques}

12 L'équipe de production s'est appropriée cinq thématiques et cinq types de dispositifs numériques.

\section{La fouille numérique ou "Strat'os"9}

13 L'idée de la fouille numérique était omniprésente dans les propositions des différentes équipes. Diverses solutions avaient été envisagées, soit virtuelles soit réelles. En parallèle, l'équipe de Science Animation de Toulouse s'était réappropriée un projet développé en open source par l'UC Davis, université Davis de Californie ${ }^{10}$, un bac à sable interactif permettant de visualiser la topographie d'un lieu. Ce dispositif semblait être la solution, mais le nouvel usage était à développer. Une journée de workshop sur ce thème avec des professionnels de la médiation venant de Paris, Toulouse et Grenoble a été mise en place sur le thème du bac à sable et de la fouille archéologique. Après avoir testé le bac, de nouveaux usages ont été inventés, un cahier des charges a été écrit et soumis à une société pour la réalisation. La fouille numérique se nomme maintenant "Strat'os" dans l'exposition Confidences d'outre-tombe, squelettes en question. Elle propose de découvrir, à l'aide de raclettes et de pinceaux, des objets virtuels enfouis dans des billes de verre.

\section{Le laboratoire d'étude du squelette ou "Profil'oscope"11}

L'anthropométrie était également un point saillant. Des dispositifs permettant de manipuler des os avaient été imaginés par toutes les équipes. Les participants du workshop voulaient être en contact le plus possible avec la vraie science, les vrais objets, les vrais scientifiques. Devant l'impossibilité de manipuler les vrais ossements et le tarif des impressions 3D, la réponse de l'équipe projet a été de numériser les squelettes en créant une table tactile d'anthropométrie sur laquelle il est possible de reconstituer un squelette sous forme de puzzle et de l'étudier (détermination de son âge, de sa taille, de son sexe) en dialoguant virtuellement par une interface de téléphone avec une vraie anthropologue, Estelle Herrsher. L'orientation scénaristique s'est donc concentrée sur ce désir de communiquer avec les vrais chercheurs, souhait relevé lors d'une étude menée par l'agence Phoebus sur les jeunes et la culture scientifique.

\section{Le scanner de maladie ou "Os-scan"12}

Lors du workshop, l'équipe Squelette Academy avait dessiné "Os-can", un scanner pour faire des diagnostics sur des squelettes et donner des informations complémentaires au résultat du scan. Le scénario de l'exposition proposait, en accord avec le comité scientifique, de mettre en scène une partie sur la paléoanthropologie. Olivier Dutour et son équipe d'anthropologues de l'équipe PACEA de Bordeaux se sont prêtés au jeu de cette forme de médiation. Ils ont fourni les contenus scientifiques de ce dispositif : film 3D montrant les effets de 3 maladies sur les os (la syphilis, la tuberculose, le myélome). 
Dans "Os'scan", à partir d'un scanner en réalité augmentée qui se déplace au-dessus d'un squelette en plastique, apparaît, selon les interrogations choisies par le visiteur, des images d'ossements lésés par de graves maladies.

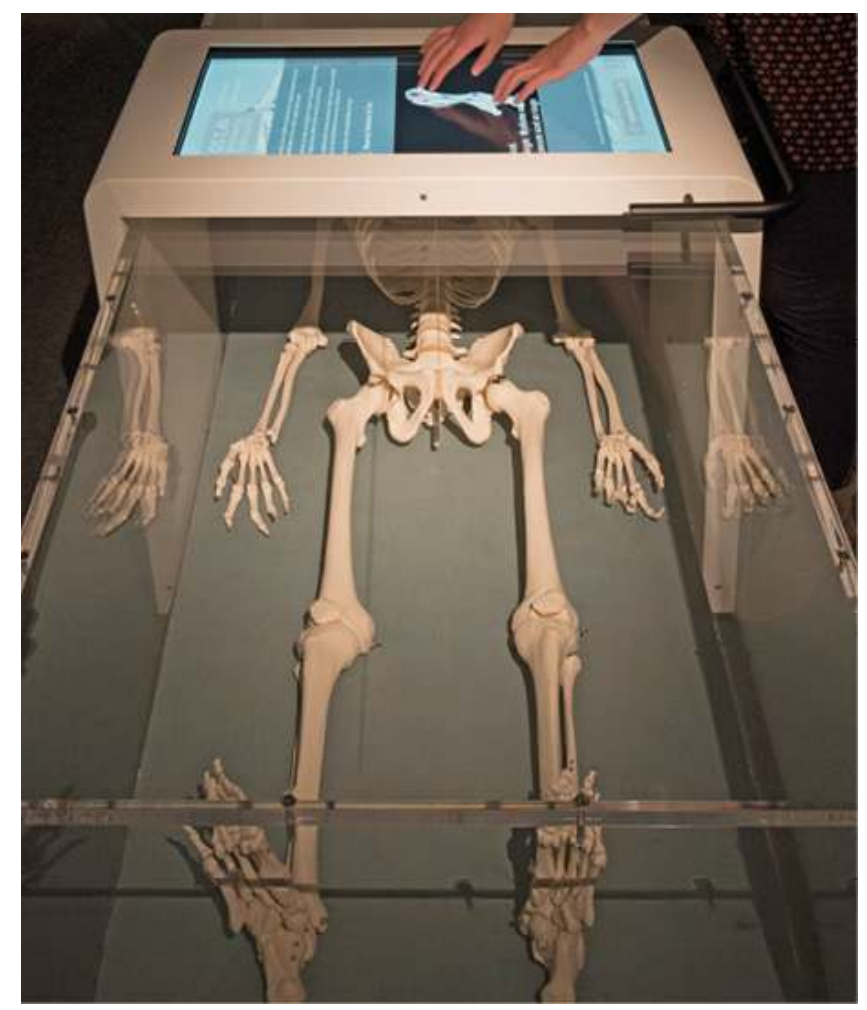

(c) Musée Dauphinois

\section{L'alimentation ou "Rest'os"13}

16 La question de l'alimentation en lien avec les restes humains était également un sujet à porter à la connaissance du public au regard des travaux originaux menés par l'anthropologue Estelle Herrsher. Dans ce cas, ce ne sont pas les participants du workshop qui ont directement fait des propositions, mais c'est plutôt l'esprit général des journées qui a été inspirant. De nombreux projets proposés tournaient autour de la réalité augmentée et des interactions homme/machine ou comment déclencher des actions entre des objets réels et des contenus numériques. L'équipe projet a donc imaginé deux réponses pour cette partie : l'une avec une vitrine écran qui permet d'augmenter des objets et de raconter leur histoire et dans ce cas précis, ce sont des mandibules humaines qui ont été choisies ; l'autre avec des puces RFID ${ }^{14}$ permettant de mettre en scène une expérience de laboratoire sur le collagène qui est un marqueur de l'alimentation.

\section{Le miroir aux squelettes ou "Mir'os"15}

Dans toutes les équipes était apparue l'idée d'interaction avec son propre squelette, sous forme de danse ou autre mouvement ludique. Bref tous les participants voulaient voir leur squelette. C'est l'équipe Osmose qui l'a dessiné. Cette idée qui peut paraître futile a été prise au sérieux par les concepteurs et un dispositif scénographié a été mis en place. "Mir'os" permet de découvrir un modèle 3D de squelette humain qui interagit avec un 
capteur de mouvement issu du monde du jeu vidéo Kinect avec le visiteur ${ }^{16}$. Ce dernier est invité à danser sur quatre compositions en lien avec la mort allant de la danse macabre de Camille Saint-Saëns à la mort sur le dancefloor de Vitalic.

\section{Un premier bilan de l'expérience}

18 À l'ère de la co-conception, cette expérience montre comment les usagers d'un musée peuvent être interrogés et inclus dans le dispositif de production d'une exposition. Pour preuve, ils sont cités dans le générique de l'exposition au même titre que le comité scientifique et tous les contributeurs. La parole qui leur est proposée est prise au sérieux par les producteurs et permet de renouveler les manières de mettre en scène les savoirs plus en lien avec leurs attentes. Ils bousculent les manières de penser et enrichissent les méthodes de travail tant du côté des participants que de celui des concepteurs de l'exposition. Une documentation précise de ce travail est également disponible sur le blog de l'exposition : Digital bones ${ }^{17}$.

Les manipulations, dispositifs, installations numériques posent question quant à leur fonction précise dans l'exposition. Ils sont des outils de transmission des savoirs que le visiteur manipule, ils proposent une expérience immersive forte, brouillent les pistes entre le réel et le virtuel. Ils sont également des objets éloignés des bornes interactives que l'on pouvait trouver dans les musées il y a quelques années. Les écrans disparaissent et laissent place à des interfaces tangibles qui ont des noms que l'on peut s'approprier.

La question des usages du numérique au cœur d'un dispositif scénographique est ainsi toujours d'actualité et les tests et études d'usage ergonomique ont une importance majeure pour les faire évoluer. Les méthodes déployées par les Living Labs allant de l'idéation aux tests sont cruciales pour perfectionner ces expériences destinées à compléter et faciliter la diffusion des savoirs.

\section{Le partenariat est-il la condition SINE QUA NON pour une médiation numérique au musée?}

21 Située à la croisée des sciences, de l'archéologie, de la sociologie et de l'art, l'exposition Confidences d'outre-tombe, squelettes en question abordait la thématique de l'archéologie funéraire et interrogeait le public sur la relation que nous entretenons avec la mort. Déployée sur près de $800 \mathrm{~m}^{2}$, la scénographie présentait des ossements humains, des documents issus de collections publiques et privées, des entretiens de professionnels (tatoueurs, musiciens...) et de particuliers (organisateur de la Zombie Walk, fans de jeux vidéo...) et des objets collectés auprès d'un large public (la Collecte de la mort), des reportages vidéos réalisés à l'occasion de l'exposition (les Paroles d'experts), des fictions sonores interprétées par des comédiens (les confidences de nos grands ancêtres) et enfin des créations artistiques commandées auprès de quarante-deux plasticiens (l'installation [K]rânes42, la catacombe artistique).

22 Si le recours à ces multiples formes d'expôts est habituel dans l'écriture des expositions au musée Dauphinois, l'intégration des dispositifs numériques riches et sophistiqués imaginés par La Casemate et ses publics revêt un caractère exceptionnel. Cette expérience pose la question des enjeux du partenariat, garant de la présence de la médiation 
numérique au musée et soulève aussi la problématique de l'efficience de l'intégration des dispositifs en termes scénographiques.

Des dispositifs numériques plébiscités par les visiteurs comme ici le "Rest'os", un espace de laboratoire pour comprendre ce que les os disent de l'alimentation et proposant des manipulations sur les thèmes de la détermination de l'alimentation en protéines à partir de l'étude du collagène des os.

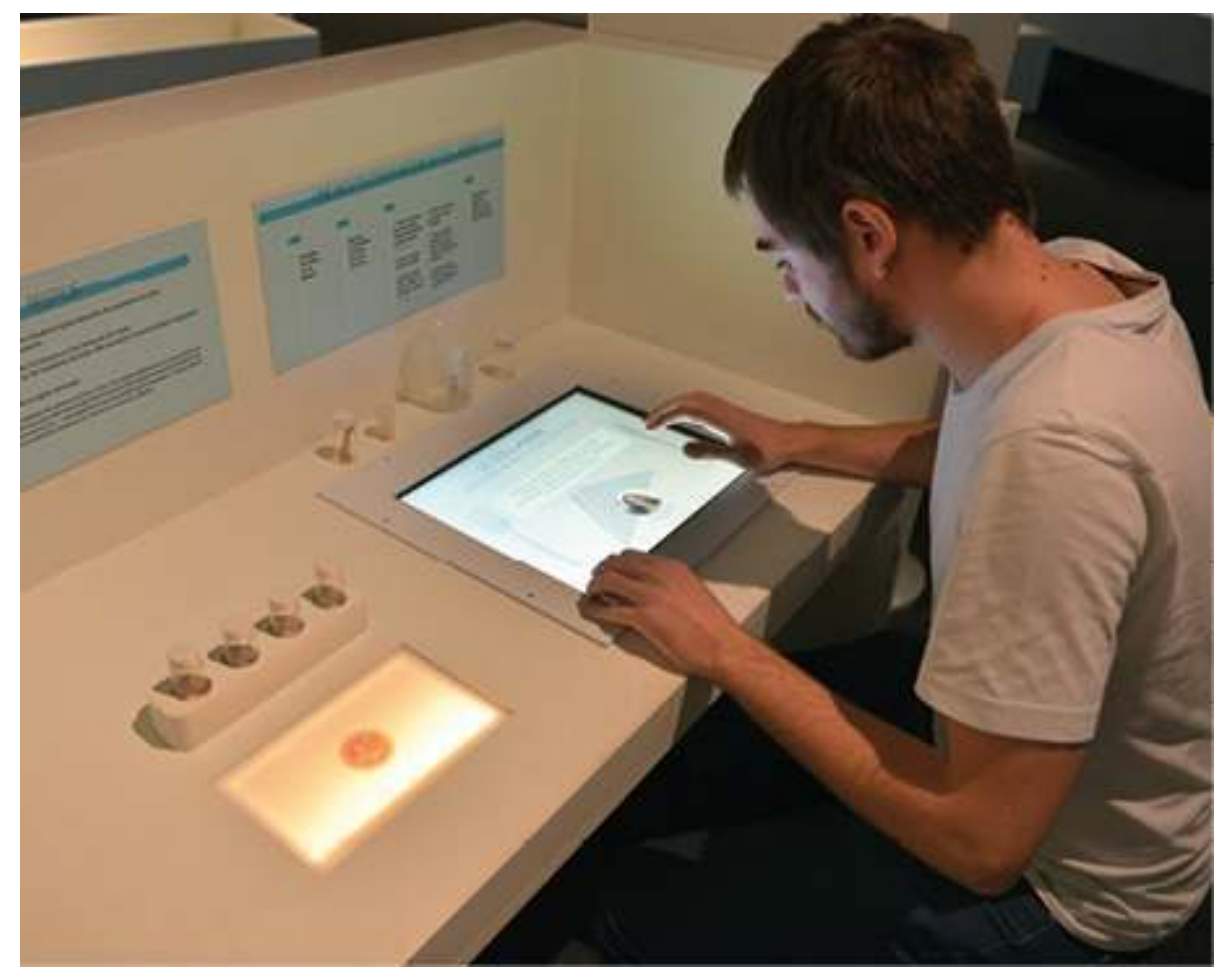

(c) Musée Dauphinois

Des projets remarquables de valorisation numérique du patrimoine, observés en France et à l'étranger, attisent l'envie de l'équipe du musée d'offrir au public des expériences inédites de visites. Les installations multimédia interactives, immersives et sensibles, les web-documentaires, les serious games, les plateformes collaboratives, les films d'animation et la réalité virtuelle offrent de nouvelles formes de narration au service des expositions. À ce jour, les expériences menées au musée Dauphinois se limitent au développement de bornes interactives pour donner accès à des corpus de collections. Une borne de conception plus élaborée, ouvrant à une fonction contributive in situ du public, est en cours de réalisation. Elle sera sous peu révélée au public dans l'exposition permanente Gens de l'alpe. Elle émane d'un prototype imaginé trois ans plus tôt, lors des rencontres Museomix organisées au musée Dauphinois à Grenoble. De façon générale, ces investissements intellectuels, technologiques et financiers sont mobilisés pour les parcours permanents et les projets à long terme, comme le démontre la plupart des projets "innovation" engagés par les musées départementaux et la Direction de la culture et du patrimoine de l'Isère (le musée Dauphinois est un service de la Direction de la culture et du patrimoine du Département de l'Isère).

La question de la présence de la médiation numérique dans les expositions temporaires se pose différemment, car la courte durée de préparation du projet demande une forte réactivité. Une exposition implique plusieurs "écritures" : l'expographie elle-même, mais 
aussi la publication, le programme de médiation, la communication. La médiation numérique constitue une écriture supplémentaire, exigeante en termes de contenus et spécifique à la dimension numérique du média. L'équipe du musée, expérimentée sur les premières formes d'écritures, est aujourd'hui encore sans profils métiers affirmés dans les domaines du numérique. L'autre facteur limitant l'expérience du numérique réside bien sûr dans la dimension technologique. Motiver le déploiement d'outils numériques innovants (en termes de logiciels et de matériels), communicants et ouverts sur les réseaux sociaux pour le temps éphémère d'une exposition temporaire, demande au porteur de projet des arguments, une anticipation et une motivation sans faille pour espérer une prise en compte par la collectivité, qui doit garantir une cohérence de son système d'information. Ce n'est pas impossible, mais...

Le partenariat établi avec La Casemate révèle une alliance "symbiotique" et une alternative efficiente au profit d'un projet commun devant s'exprimer le temps d'une exposition temporaire. La médiation numérique est une forme fondamentale du projet culturel du CCSTI et une pratique affirmée depuis plusieurs années. Les échanges avec les chercheurs, les ateliers participatifs avec le public, la complicité avec les conservateurs des musées, les relations étroites avec les sociétés de réalisation multimédia, ont permis de produire aisément ces outils de médiation numérique. Leur intégration dans le parcours scénographique fut pensée dès le début du projet avec les commissaires et le scénographe. Les dispositifs offraient des accès variés et divertissants au savoir. Le financement d'un tel projet de médiation numérique, que le musée n'aurait pu assumer seul, a bénéficié de l'aide du programme Inmédiats, dans lequel La Casemate est acteur. Un public inhabituel a fréquenté l'exposition : des jeunes entre 15 et 25 ans, des familles avec de très jeunes enfants... Plus de 65000 personnes ont visité et apprécié la complémentarité des approches. Une étude d'évaluation, menée par la société Multicom à Meylan (Isère) a montré que le public manipulait les dispositifs sans négliger l'observation des expôts et même la lecture des textes de l'exposition. Le retour à une forme expographique plus "traditionnelle" doit être négocié car la médiation numérique est de plus en plus réclamée.

Cette expérience de partenariat questionne sur la vocation du musée : doit-il être luimême producteur de l'innovation numérique ? L'équipe est-elle en mesure de se spécialiser dans ces domaines en constante mutation? Ou bien le musée doit-il privilégier sa participation à des projets conçus par des acteurs extérieurs ? Doit-il interpeller des créateurs? La réponse actuellement la plus pertinente pour enrichir les expositions est de privilégier les partenariats avec des acteurs spécialistes de la médiation et de la création numériques.

Franck Philippeaux

8 Co-commissaire de l'exposition Confidence d'outre-tombe, squelettes en question, Conservateur du patrimoine au musée Dauphinois

\section{L'interactivité numérique au service de l'archéométrie}


La table tactile d'anthropologie "Profil'oscope" permettait aux visiteurs une reconnaissance rapide des ossements humains en proposant un jeu interactif pour déterminer l'âge, la taille et le sexe de trois individus

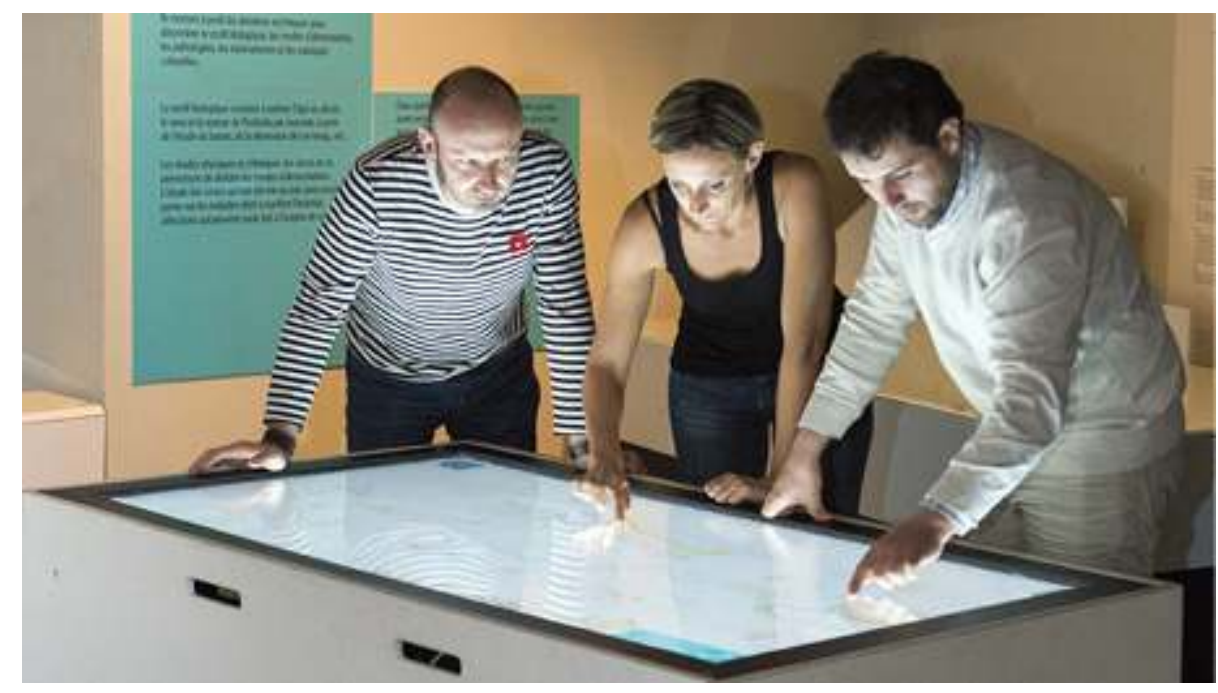

() Musée Dauphinois plus d'archéologie, c'est celui de l'archéométrie. Cette discipline, ou plutôt des domaines techniques autour de plusieurs disciplines scientifiques, renvoient à la question légitime du public quand il découvre des sujets liés à des périodes anciennes : comment le sait-on ?

Dans le cas du musée archéologique Grenoble Saint-Laurent, musée de site traitant des premières églises et des premiers cimetières de Grenoble, les fouilles conduites par Renée Colardelle de 1978 à 1998, ont permis l'exhumation de 1503 sépultures, soit avec les phénomènes de ré inhumations, près de 1800 squelettes allant du IVe siècle jusqu'au XVIIIe siècle. Rares sont les occasions d'étudier l'évolution d'une population urbaine sur une période aussi longue. Aussi le fond anthropologique de Saint-Laurent fit l'objet de recherches de pointe, et notamment dans le domaine de la biologie des ossements et de la tracéologie dentaire avec une équipe d'anthropologues du CNRS et de l'université de Bordeaux animée par Estelle Herrscher. Initié par la Casemate et le musée archéologique Grenoble Saint-Laurent, le projet d'exposition autour des investigations anthropologiques des squelettes de Saint-Laurent, a pris de l'ampleur dans tous les champs de l'anthropologie culturelle et de l'histoire de ses représentations avec le pilotage et la réalisation de l'exposition au musée Dauphinois.

Des dispositifs inventés par La Casemate en collaboration étroite avec les chercheurs, je retiendrai particulièrement "Profil'oscope", "Os'scan" et "Rest'os" sur les cinq présentés dans l'exposition Confidences d'outre-tombe, squelettes en question. En somme, bien qu'il reste toujours très difficile de rendre compte auprès du public des résultats d'analyses de laboratoire, ces outils d'animation numérique conçus, pratiques et didactiques, ont été globalement très appréciés dans leur appréhension et leur utilisation. Ils contribuèrent non seulement à proposer des pauses dans un environnement studieux et réfléchi sur un sujet grave, mais à rendre le visiteur actif en lui proposant le statut nouveau d'apprenti expert. 

Casemate ont été un point fort de l'exposition et une clé de compréhension de notions difficiles du profil des individus par les squelettes, de la pathologie ou des traumatismes de certains d'entre eux, donnant une image différente des restes inertes présentés tout autour dans une sorte de "salle blanche" (la seule animation qui a été conçue par le musée Dauphinois fut celle des confidences racontées, fictives mais toujours documentées par la période d'appartenance, l'archéologie du contexte local, les observations anthropologiques de personnages sans nom et sans histoire connue).

Enfin, cette collaboration institutionnelle nouvelle a offert la possibilité au musée archéologique de Grenoble d'accéder, pour des problématiques scientifiques non résolues, à un équipement scientifique de prestige - l'ESRF (European Synchrotron Radiation Facility) - avec un chercheur, Paul Tafforeau, qui a développé un système de lecture thomographique permettant de lire et d'identifier trois médailles en terre cuite agglomérées dans une boîte en fer, retrouvée dans une tombe du XVIIe siècle et qui ne pouvait être ouverte par un système de restauration classique au risque de tout détruire : les résultats de cette étude ont été présentés dans l'exposition.

\section{Résultat de l'évaluation des dispositifs numériques}

\section{Le puzzle squelettique du "Profil'oscope"}

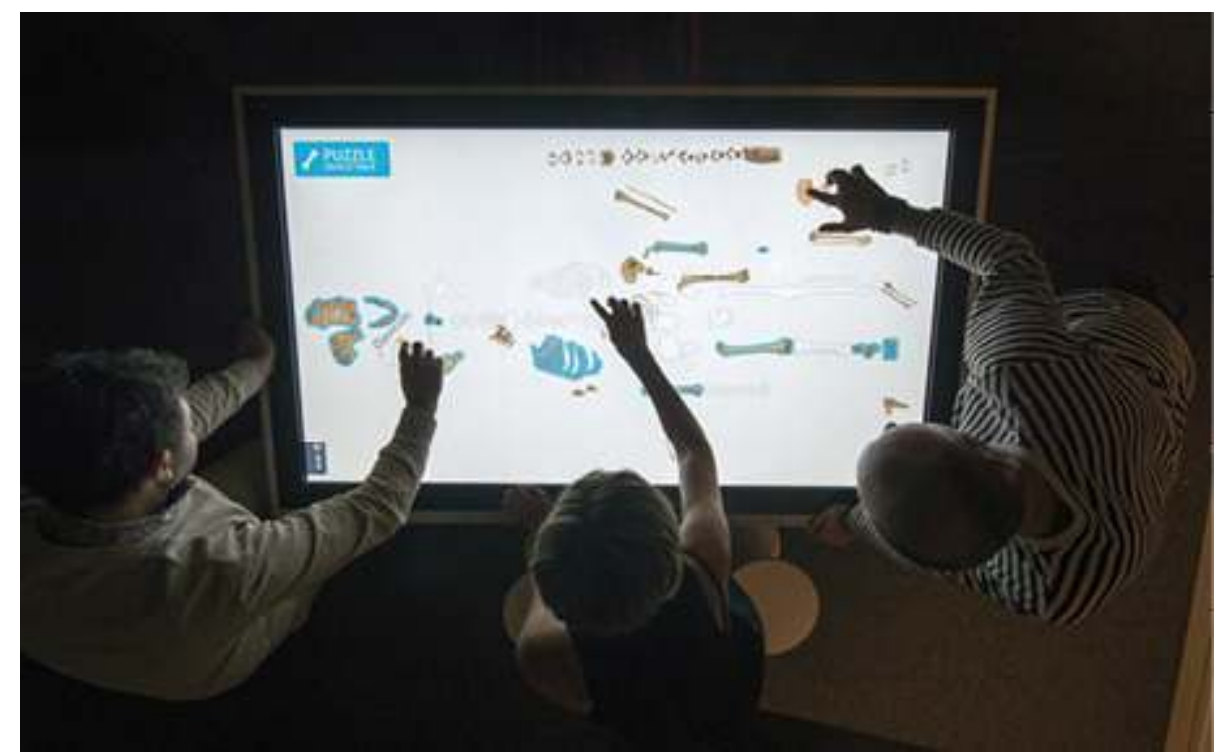

(C) Musée Dauphinois

Trois des cinq dispositifs - "Strat'os", "Profil'oscope" et "Os'scan" ont été évalués avec des utilisateurs par la société Multicom. Les tests ont été réalisés auprès de 21 personnes. Les participants ont été contactés via la base de données de participants de Multicom et invités à répondre à un questionnaire de pré-recrutement, nous permettant de contrôler 
certaines variables : tous les participants ont un niveau d'étude équivalent (autour de Bac +5) et des compétences relatives à l'utilisation des nouvelles technologies.

Le test se déroulait de la façon suivante : chaque participant était accueilli à l'entrée du musée, conduit devant l'exposition, puis l'expérimentateur lui énonçait les consignes, lui demandant d'agir avec chaque dispositif comme il le ferait naturellement. Le participant était filmé pendant toute la durée de son interaction. À la suite de l'interaction avec le dispositif, un entretien de débriefing était réalisé avec le participant sur sa compréhension du dispositif, les difficultés rencontrées, les points positifs... Enfin, le participant était prévenu de toutes les méthodes de recueil de données.

À titre d'exemple, nous présentons ici l'évaluation du dispositif "Profil'oscope".

39 Le puzzle : $85 \%$ des utilisateurs ont réussi à compléter le puzzle. Les principales difficultés identifiées concernent la compréhension de la consigne qui n'indique pas que les os se déplacent et peuvent pivoter tactilement. Elle ne précise pas non plus que la surbrillance bleue indique qu'un os est à sa place.

Le jeu de la détermination de l'âge d'un enfant : $25 \%$ des utilisateurs ont réussi ce jeu. Les principales difficultés identifiées concernent les indications données aux utilisateurs pour résoudre ce jeu : dans un exemple donné, la mâchoire cible compte 3 molaires, or la bonne réponse est une mâchoire comptant 4 molaires. Lorsque l'utilisateur se trompe, il peut consulter la bonne réponse pour comprendre son erreur mais la réponse proposée n'explique pas la méthode opérée pour trouver la bonne réponse.

41 Le jeu de la détermination de la taille : $85 \%$ des utilisateurs ont réussi ce jeu. Les utilisateurs n'ont pas rencontré de difficulté pour utiliser ce jeu.

Le jeu de la détermination du sexe : $50 \%$ des utilisateurs ont réussi ce jeu. Les principales difficultés identifiées concernent le déplacement et la modification de la taille des patrons pour réussir ce jeu. Seule la moitié des utilisateurs ont utilisé ces deux fonctions. Les consignes invitent l'utilisateur à "regarder" mais pas à "toucher". L'utilisateur n'est pas suffisamment incité à utiliser les fonctions tactiles. La correspondance des formes n'est pas évidente : aucun des deux patrons ne correspond exactement à la forme de l'échancrure de l'os cible.

Recommandations :

44 - les utilisateurs ont parfois des difficultés pour accéder aux jeux et semblent perdus sur l'écran intermédiaire : conserver un écran intermédiaire proposant des informations sur l'expérience, mais supprimer l'écran affichant le squelette, de façon à accéder au jeu directement après avoir fermé les informations.

45 - les utilisateurs se trompent beaucoup pour le jeu de la détermination de l'âge d'un enfant : changer les modèles de mâchoires et la mâchoire à étudier de façon à éviter les pièges pour les utilisateurs. Puisque la méthode pour déterminer l'âge consiste à compter les molaires, alors le nombre de molaires de l'os cible doit être égal au nombre de molaires de l'os correspondant à la bonne réponse. Anne Pellegrin

Docteur en Sciences cognitives, ergonome et manager de la business unit Multicom de Floralis 


\section{NOTES}

1. Livret Acte\#3 des Ateliers Inmédiats.

2. Médiamétrie / Netratings, L'audience des 50 premiers groupes en France en septembre 2014.

3. Duclos, J.-C. La co-écriture au musée. La Documentation française, 2011, pp. 111-118.

4. www.openlivinglabs.eu/

5. www.museomix.org/

6. www.youtube.com/watch?v=vwFETkov-2o

7. www.echosciences-grenoble.fr/actualites/

8. www.youtube.com/watch?v=Q-x0Nhl6vdA

9. www.youtube.com/watch?v=hoixk93-RAc

10. http://idav.ucdavis.edu/ okreylos/ResDev/SARndbox/

11. www.youtube.com/watch? $v=\mathrm{Kw}$-umrNHMdA

12. www.youtube.com/watch? $v=G j X c V o d j Q p k$

13. www.youtube.com/watch? $\mathrm{v}=68 \mathrm{SFCP} 4 \mathrm{hBqQ}$

14. https://fr.wikipedia.org/wiki/Radio-identification

15. www.youtube.com/watch?v=peZKJvY0t3I

16. http://fr.wikipedia.org/wiki/Kinect

17. www.echosciences-grenoble.fr/sites/digital-bones

\section{RÉSUMÉS}

Ce retour sur une expérience de co-construction d'une exposition avec des visiteurs montre comment les usagers d'une institution muséale peuvent être interrogés et associés au dispositif de production et comment les réseaux sociaux permettent de renouveler le dialogue ainsi instauré et inspirer la mise place de nouveaux dispositifs numériques.

\section{INDEX}

Mots-clés : dispositif numérique

\section{AUTEUR}

\section{LUDOVIC MAGGIONI}

responsable des expositions à La Casemate à Grenoble, professeur associé à l'université Stendhal de Grenoble

ludovic.maggioni@lacasemate.fr 